\title{
DESCENT TRAJECTORY MODELLING FOR THE LANDING SYSTEM PROTOTYPE
}

\author{
Henrich GLASER-OPITZ ${ }^{*}$, Ján LABUN ${ }^{2}$, Kristína BUDAJOVÁ ${ }^{3}$, Leonard GLASER-OPITZ ${ }^{4}$ \\ 1,2,3Faculty of Aeronautics, Technical University of Košice, Slovakia \\ ${ }^{4}$ Slovak Republic Air Traffic Services SR (LPS SR š. p.), Bratislava, Slovakia
}

Received 3 August 2016; revised 20 March 2017; accepted 8 April 2017

\begin{abstract}
This paper gives another view on a method used for aircraft approach and landing phase of flight that enables replacement of standard glideslope. Proposed Landing System is based on Terrain Reference Navigation (TRN) using own created terrain elevation database, based on Radar Altimeter (RA) measurements compared to the overflown terrain. Simulations were performed on a chosen airport (KSC - Košice Airport) and aircraft (Boeing 737-800), where descend procedures was designed based on real airline data in compliance with Initial 4D Trajectory (i4D). Descend trajectory was modelled with EUROCONTROL Base of Aircraft DAta (BADA) performance model as a Continuous Descent Approach (CDA) from proposed merging point to the KSC RunWaY (RWY) threshold. This method was proposed to enhance pilot situational awareness in situations when standard Instrument Landing System (ILS) information could be lost or misleading and without the need of any ground station for successful navigation and guidance to the RWY threshold. Landing System prototype flight test were performed on full mission flight simulator.
\end{abstract}

Keywords: terrain relative navigation (TRN), landing system, base of aircraft data (BADA), continuous descent approach (CDA), initial 4D trajectory (i4D), full mission flight simulator.

\section{Notations}

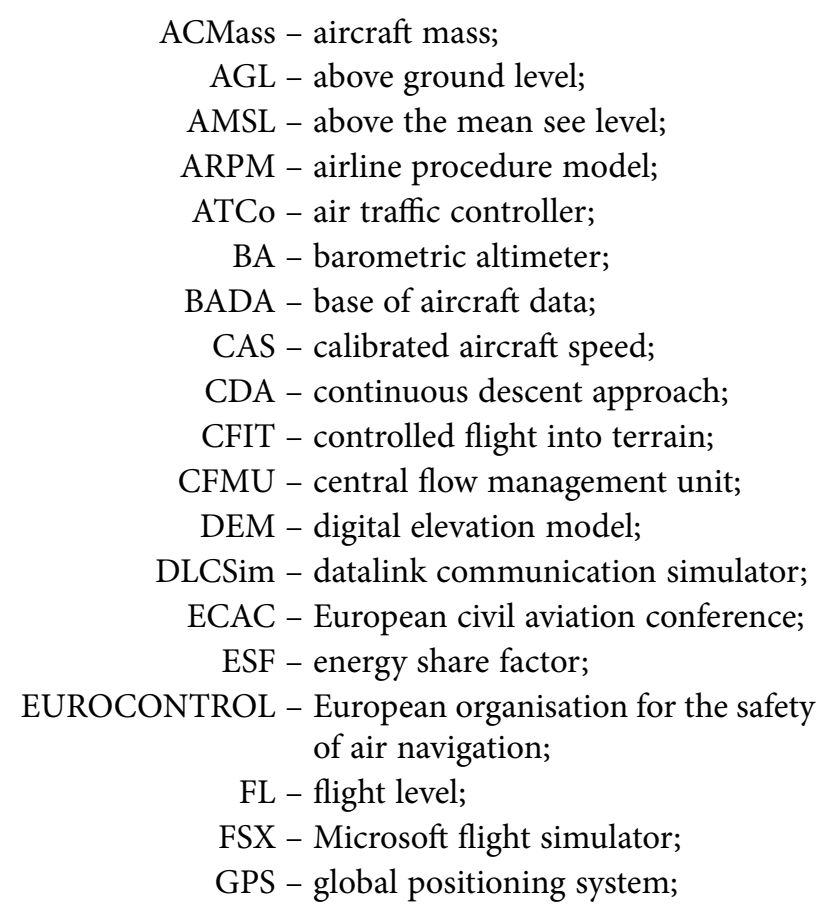

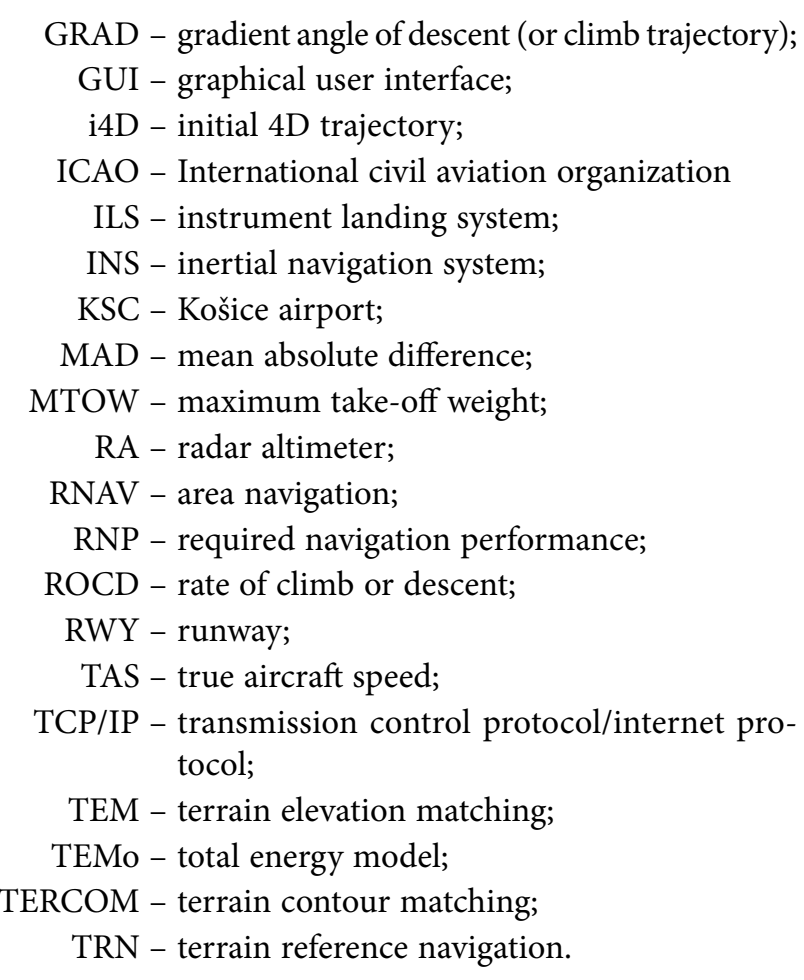

${ }^{*}$ Corresponding author. E-mail: henrich.glaser-opitz@eurocontrol.int 


\section{Introduction}

The continuing growth of aviation increases demands on airspace capacity therefore emphasizing the need for optimum utilization of available airspace. It is expected that all future RNAV/RNP applications will identify the navigation requirements through the use of performance specifications rather than defining equipage of specific navigation sensors (ICAO 2013).

This paper describes a work where the final phase of flight - descent and approach to the destination airport could be done more efficiently and safe using performance based navigation principles. The flight safety is one of the most important features in aviation. In recent years several times occurred accidents or incidents that took place due to not mechanical failure or maintenance malfunction, but due to pilot CFIT, caused by the loss of pilots situational awareness. CFIT includes among the collision with the ground at low altitudes, also aircraft impacting hillside and mountainsides while at higher altitudes, even thousands of feet above mean see level. The current initiative is to improve aviation safety, with a primary focus on reducing CFIT. This can be achieved with integration of digital terrain database into TRN and other on-board navigation data (Meduna 2011). The output of this paper is directly applicable on the CFIT accidents reduction by vertical position uncertainty reduction increasing the pilot situational awareness. TRN together with digital terrain database has the potential for vertical channel accuracy improvements during the approach and landing procedure for both civil and military applications. Terrain profile knowledge enables integration of RA into the navigation solution. The TRN implementation is completed with simulations using created digital terrain database. Descent trajectory used in developed Landing System is modelled using EUROCONTROL BADA performance model. This trajectory is designed for a specific airport (KSC) in compliance with i4D trajectory and then modelled and implemented into the Landing System. The Landing System was designed to be able to interact with FSX/Prepar3D flight simulator and to communicate with developed DLCSim (Glaser-Opitz, H., Glaser-Opitz, L. 2015b; Glaser-Opitz et al. 2015). It increases mutual interaction between pilot and ATCo and improves procedures for pilot and controller testing (e.g. workload) and training.

\section{TRN}

The main goal of the TEM algorithm is to fuse the information from RA, terrain database, INS and BA measurements. The data output fusion should represent the estimation of the aircraft position together with an estimation error. There exist many ways how to approach this fusion problem. We have chosen the batch correlation TRN concept. The idea is that by collecting a large enough number of measurements, the TRN likelihood surface will collapse to a near-unimodal distribution. This allows position to be uniquely determined from the measurements (ICAO 2013).

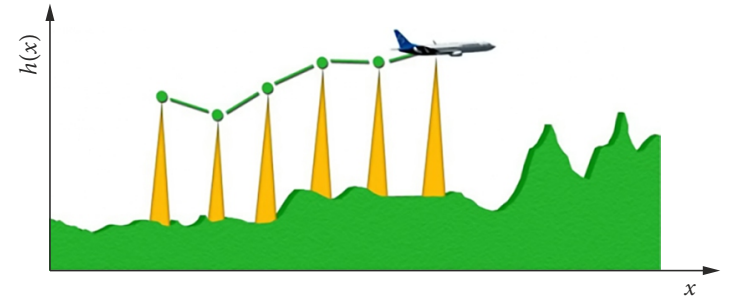

Figure 1. Batch correlation TRN concept (TERCOM)

Figure 1 depicts the process of an aircraft collecting a series of RA measurements along its path, and correlates this resulting measured profile to generate a position estimate. A technique used for position determination is called TERCOM. Several simulations were made using MATLAB $^{\circledR}$ (https://www.mathworks.com) implementing TERCOM algorithm and using own created terrain elevation database. Because during take-off or landing phase we expect the curved flight trajectory, the example simulation is concentrated on this case. This flight path consists from 30 samples of elevation data with resolution same as the DEM grid, meaning $900 \mathrm{~m}$ long flight path in a rough environment (with higher elevation gradient). Elevation samples are in this case noiseless. Such situation is illustrated in Figure 2.

The blue cross in Figure 3 indicates the estimated position counted by TRN. From comparison with straight flight path simulation one can see, that we better eliminated the uncertainty of estimated position. This happened because the elevation profile of simulated flight path (Figure 4) is even more variable, what leads to smaller amount of possible positions of the aircraft according to the DEM data.

Uncertainty of estimated position due to the smooth terrain under the aircraft can be eliminated by acquiring more data from RA, using DEM with greater resolution $(10 \mathrm{~m})$ and using INS or GPS and filtering techniques for better estimation.

\section{Descent trajectory modelling with BADA}

All simulations to validate the proposed model for trajectory simulations were performed using a specifically chosen type of aircraft. According to EUROCONTROL (2014) data shows that Boeing 737-800 (B738 - BADA designator) represents the most used aircraft in the ECAC area, based on the CFMU traffic statistics over the last 12 months, representing $15.871 \%$ off overall traffic. Based on this data B738 was chosen as a suitable representative of European fleet for model validation process, as we are mainly focused on the aircraft with MTOW $>5700 \mathrm{~kg}$, usually used for commercial flights. After the analysis of sustainability, actuality, aircraft models coverage, performance, future development options and accessibility of different models, BADA family 3 was chosen as an appropriate model. BADA 3 model was used for trajectory modelling within the Landing System to model the glideslope and aircraft descent, and approach trajectory. 


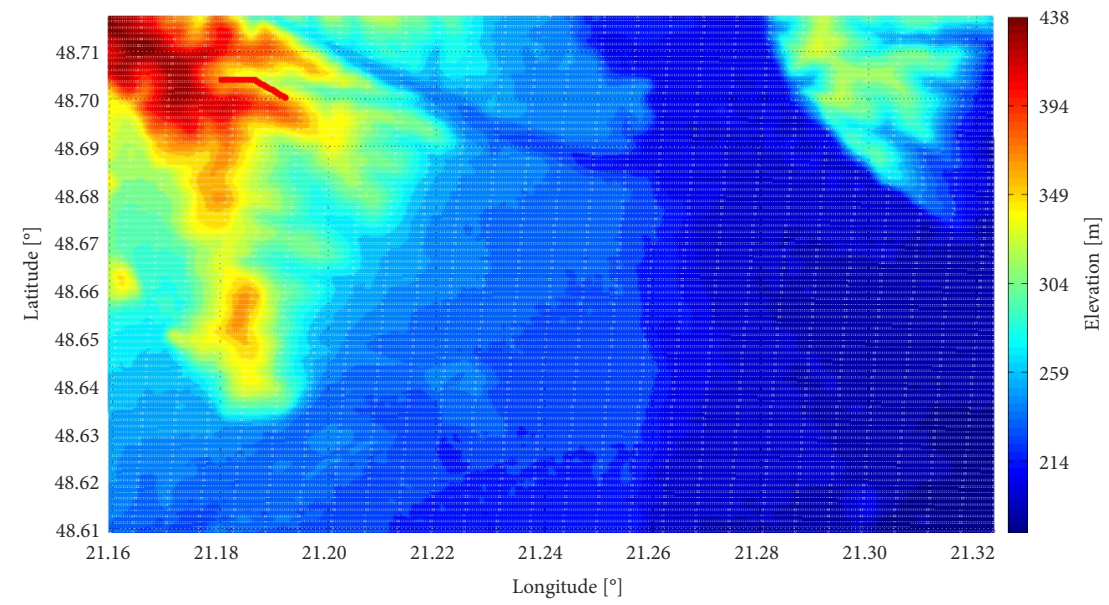

Figure 2. KSC elevation map (30 m resolution) with simulated flight trajectory (curved)

a)

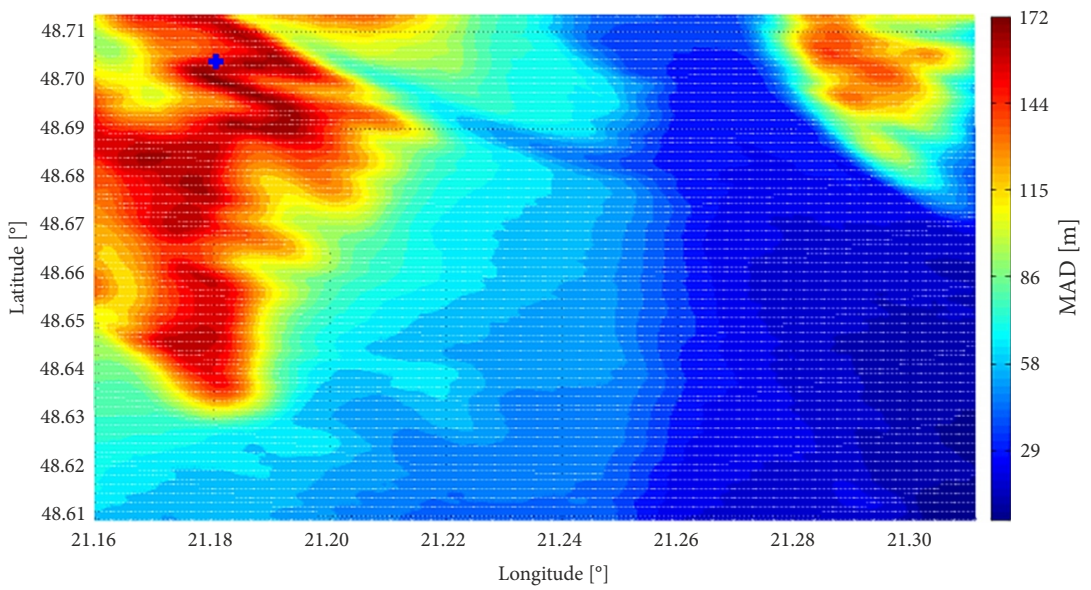

b)

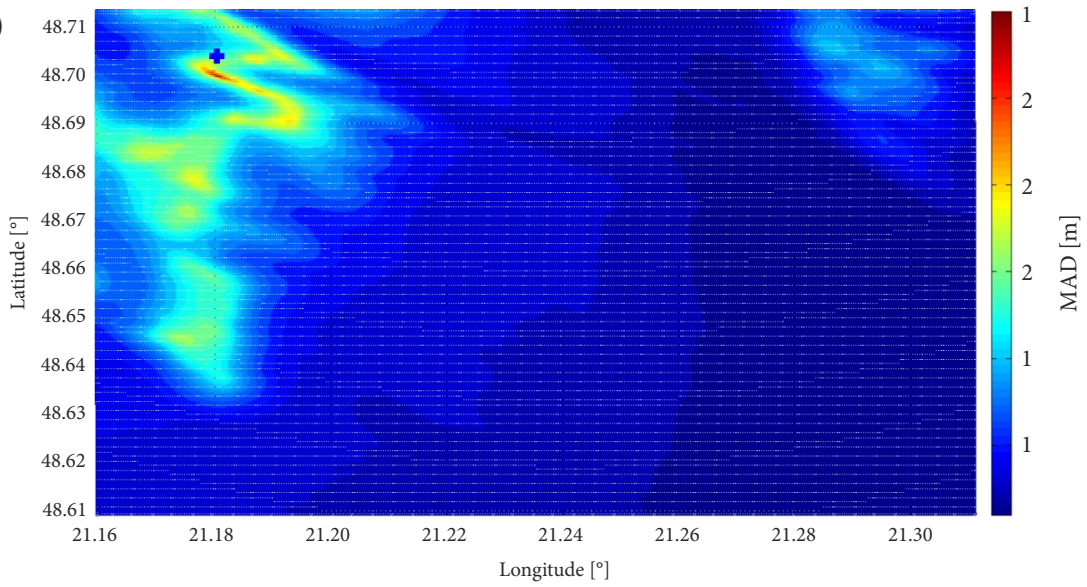

Figure 3. KSC TERCOM MAD map with calculated estimation of current position from simulated flight trajectory (curved - without noise): a - normal scale; b - logarithmic scale

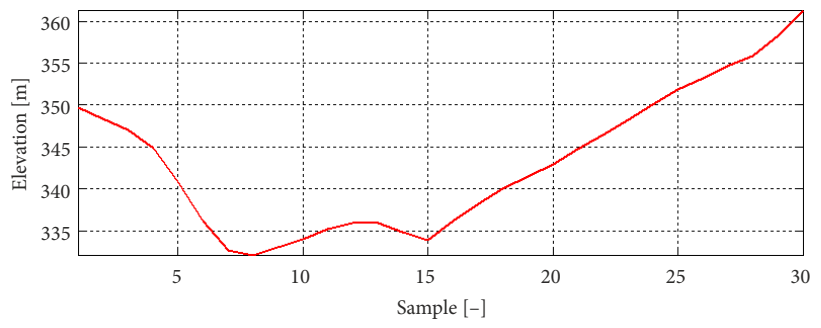

Figure 4. Elevation profile of a simulated flight trajectory (curved trajectory) 


\subsection{BADA model overview}

BADA is based on the so-called TEMo that can be considered as being a reduced point-mass model. TEMo equates the rate of work done by forces (Figure 5) acting in the aircraft to the rate of increase in potential and kinetic energy (EUROCONTROL 2015).

TEMo can be expressed by the Equation (1) as follows (EUROCONTROL 2015):

$$
(T-D) \cdot V_{T A S}=m \cdot g_{0} \cdot \frac{\mathrm{d} h}{\mathrm{~d} t}+m \cdot V_{T A S} \cdot \frac{\mathrm{d} V_{T A S}}{\mathrm{~d} t},
$$

where: $T$ is temperature; $D$ is drag; $V_{\text {TAS }}$ is actual TAS speed; $m$ is the aircraft weight; $g_{0}$ is gravitational constant; $\frac{\mathrm{d} h}{\mathrm{~d} t}$ is the change in altitude during the time sample of $\mathrm{d} t$.

Calculation the ROCD, where the speed and the throttle are controlled, is the most common situation. Such situation can be calculated using TEMo Equation (1), what can be rearranged in a way of expressing the ROCD calculation as follows:

$$
\begin{aligned}
& R O C D=\frac{T-\Delta T}{T} \cdot \frac{(T H R-D) \cdot V_{T A S}}{m \cdot g_{0}} \times \\
& 1+\left(\frac{V_{T A S}}{g_{0}}\right) \cdot\left(\frac{\mathrm{d} V_{T A S}}{\mathrm{~d} h}\right)
\end{aligned}
$$

where: $\Delta T$ is temperature deviation; THR is thrust; $D$ is drag.

As shown in EUROCONTROL (1987) and Gillet et al. (2010), equation (1) can be rewritten by introducing an ESF as a function of a Mach number $-f(M)$ :

$$
E S F=f(M)=\frac{1}{1+\left(\frac{V_{T A S}}{g_{0}}\right) \cdot\left(\frac{\mathrm{d} V_{T A S}}{\mathrm{~d} h}\right)},
$$

where: $M$ is Mach number.

This leads to:

$$
\begin{aligned}
& R O C D=\frac{\mathrm{d} h}{\mathrm{~d} t}=\frac{T-\Delta T}{T} \times \\
& \left(\frac{(T H R-D) \cdot V_{T A S}}{m \cdot g_{0}}\right) \cdot f(M) .
\end{aligned}
$$

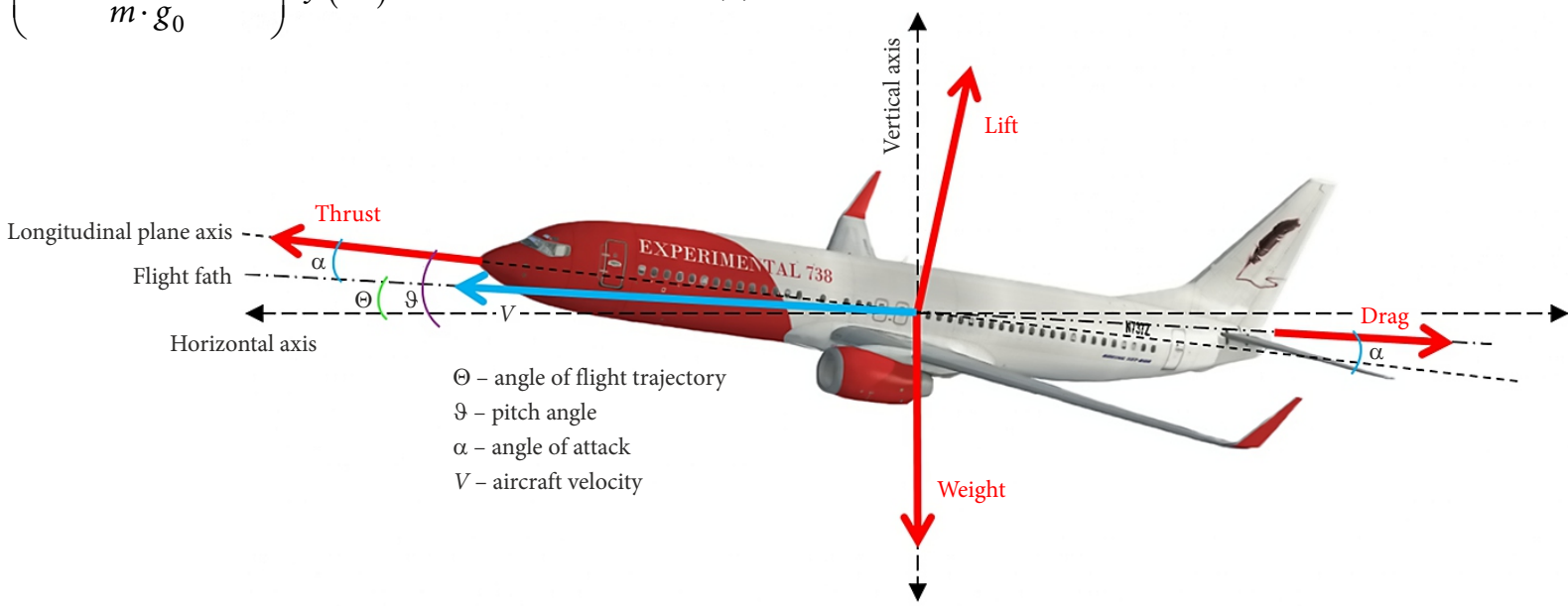

Figure 5. Aerodynamic forces acting on the aircraft (Gričová 2016)
As stated in EUROCONTROL (2015), the ESF specifies how much of the available power is allocated to climb or descent as opposed to an acceleration/deceleration while following speed profile during climb or descent. Difference between descent and climb is that the available power is negative for descent.

The change in speed in descent or climb phase can be derived from (3) as follows:

$$
\mathrm{d} V_{\text {TAS }}=\left(\frac{1}{E S F}-1\right) \cdot \frac{g_{0}}{V_{T A S}},
$$

where: $\mathrm{d} V_{T A S}$ is the change in TAS speed with chosen ESF value, which will be used for calculation of speed update for next iteration of simulation algorithm as follows:

$$
T A S_{\text {new }}=V_{T A S}+\mathrm{d} V_{\text {TAS }} \text {. }
$$

Graphical representation of calculated ROCD in descend phase of flight is illustrated in Figure 6, where we are simulating descent with B738 from FL350 to FL100 with constant speed $290 \mathrm{kt}$.

\subsection{KSC ARPM descent design}

The way an aircraft is operated differs in function of specific airspace procedures and operating policies of every airline. That is the reason why we have designed and simulated a specific procedure for aircraft descend and approach to KSC. Proposed ARPM for KSC descent and approach is shown in Figure 7.

Figure 8 depicts proposed descent procedure design based on an i4D trajectory concept. We have created a merging point for approach to both runways RWY01/19 at a distance of $29 \mathrm{NM}$ and altitude of $10000 \mathrm{ft}$. The approach follows standard approach procedures from the $5.5 \mathrm{NM}$ distance at $2500 \mathrm{ft}$ for both RWY with airport elevation of $750 \mathrm{ft}$ AMSL.

This concept allows cabin crew to follow approach route regardless to runway orientation by simply following the same designed paths for both runways. In the figure (Figure 8) could be seen three different colours. Red line is for optimized i4D trajectory concept with continuous descent from altitude $10000 \mathrm{ft}$ to airport's elevation based on BADA model. Alongside routes at each fix are displayed 
altitudes and distances from runway threshold for each route. Blue line indicates an option for direct entry for approach at each runway with indication of necessary altitude for continuous descent. Black line indicates original route designed for approach at KSC.

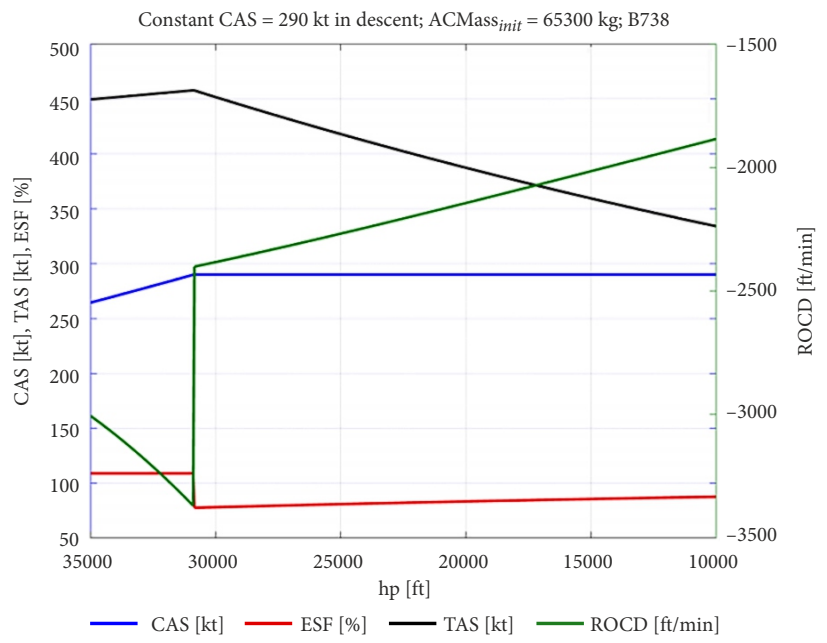

Figure 6. Descending with constant CAS for B738
When we compare blue numbers to black ones, which indicates the original requested altitudes for airplanes approach, lower values could be seen, what leads to lower speed, higher required engine thrust and higher fuel consumption, caused by maintaining lower altitudes.

Proposed descent procedure for KSC descent trajectory (Figure 8) was designed using BADA model for later use in developed Landing System. Figure 9 illustrates a top view descent trajectory initiating from proposed merging point to RWY01 KSC. The whole procedure is designed as a CDA with $3^{\circ}$ angle, what means approximately $29 \mathrm{NM}$ long path.

The advantage of such modelling is that we now have the great amount of information for modelled trajectory to be used in developed Landing System for better aircraft position and movement prediction. Data available (Figure 10) includes not only GPS coordinates and altitude, but also other important information, e.g., desired aircraft speed corresponding to defined ARPM and many more.

In depth analysis included descent procedure simulations while changing ESF values together with ACMass. Figure 11 depicts just an example of the different ESF values impact on descent procedure.

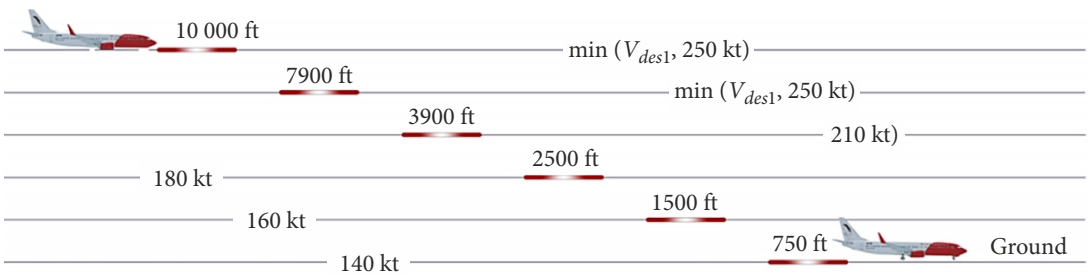

Figure 7. Descent ARPM design for Jet aircraft for KSC

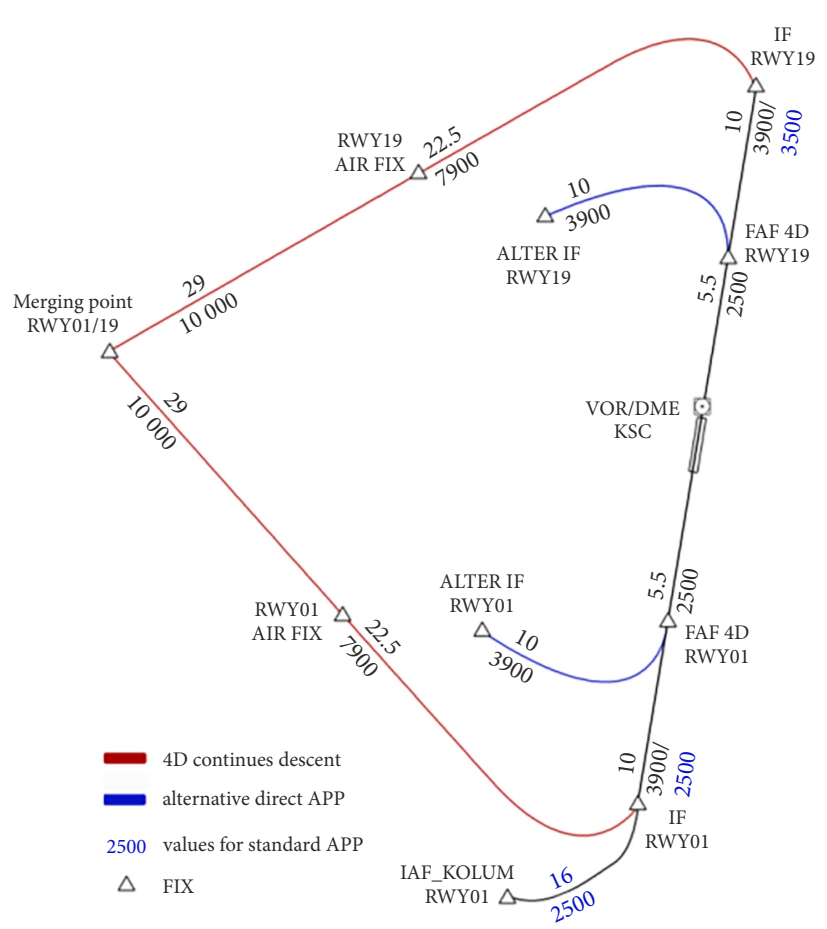

Figure 8 . KSC $4 \mathrm{D}$ trajectory descent procedure design

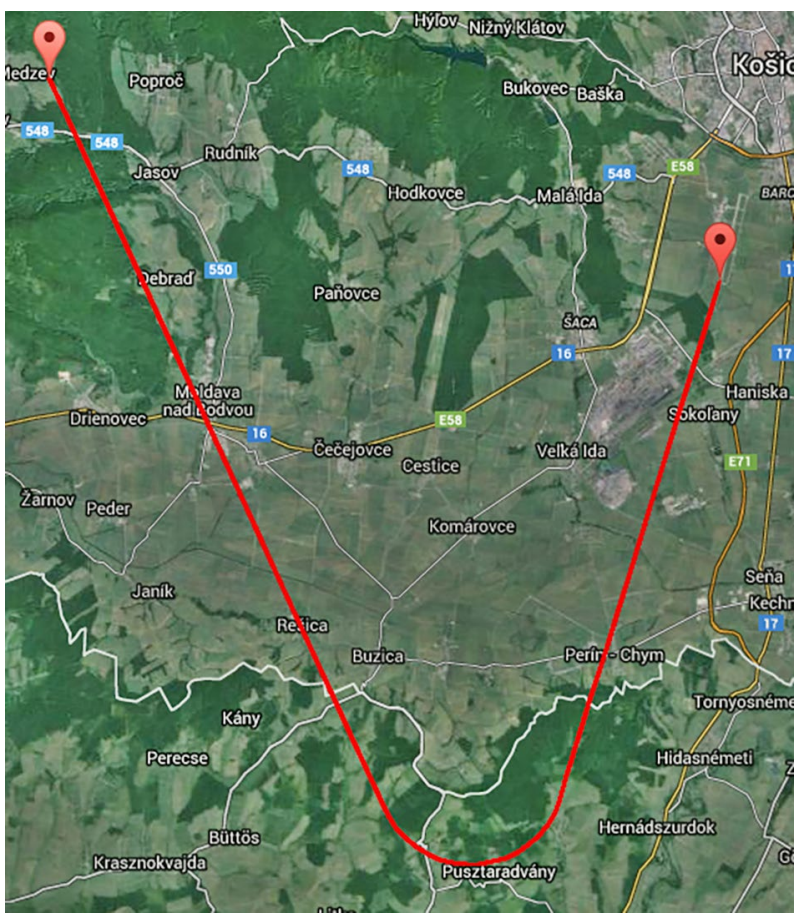

Figure 9. Proposed KSC descent trajectory modelled using BADA 


\begin{tabular}{|c|c|c|c|c|c|c|c|c|c|c|c|c|c|c|c|c|c|}
\hline$\Delta$ & A & B & c & D & E & $\mathrm{F}$ & G & $\mathrm{H}$ & 1 & $k$ & L & $M$ & $\mathrm{~N}$ & 0 & $p$ & Q & $R$ \\
\hline 1 & $\mathrm{Hp}[\mathrm{ft}]$ & ACMass[kg] & CAS [kt] & TAS[kt] & $\mathrm{M}[-]$ & ROCD $[\mathrm{ft} / \mathrm{min}]$ & Grad[deg] & Fuelflow $[\mathrm{kg} / \mathrm{s}]$ & ] Fuel[kg] & Time[s] Distance[NM] & ] $\operatorname{Thr}[\mathrm{N}]$ & $D[N]$ & $\mathrm{fM}[-]$ & bankAngle $\left[{ }^{\circ}\right]$ & $\left.{ }^{\circ}\right]$ bearing["] & LAT[ $\left.{ }^{\circ}\right]$ & LON [0] \\
\hline 2 & 10000 & 65300 & 250 & 288.7023134 & 0.452275114 & -1532.218816 & & 3 1.196718817 & 1.1967188 & 0 & 080683.95419 & 43463.68775 & 0.9016752 & & 0155.3397492 & 48.7118 & 20.92515 \\
\hline 3 & 9974.46302 & 65298.80328 & 250 & 2 288.5934003 & 0.4520618779 & -1531.640786 & & 31.196572139 & 2.3932909 & 10.0801950870 & of 80680.54136 & 43464.20175 & 0.9017538 & & 0155.3403838 & 48.71058613 & 20.92599456 \\
\hline 4 & 9948.935673 & 65297.60671 & 250 & 288.4845838 & 0.4518488534 & -1531.063268 & & 31.196425604 & 3.5897165 & 20.1603599205 & 580677.13193 & 43464.71458 & 0.9018323 & & 0155.3410182 & 48.70937271 & 20.92683877 \\
\hline 5 & 9923.417952 & 65296.41028 & 250 & 288.3758637 & 0.45163604 & -1530.486262 & & 31.196279213 & 4.7859957 & 3 0.2404945271 & 180673.72591 & 43465.22623 & 0.9019107 & & 0155.3416522 & 48.70815974 & 20.92768261 \\
\hline 7 & 9872.411352 & 65294.01787 & 250 & 2 288.1587122 & 0.4512110455 & -1529.333782 & & 31.195986861 & 7.1781155 & 50.400673167 & 80666.92405 & 43466.24603 & 0.9020673 & & 0155.3429194 & 48.70573516 & 20.92936923 \\
\hline 8 & 9846.922455 & 65292.82188 & 250 & 288.0502806 & 0.4509988639 & -1528.758307 & & 31.195840899 & 8.3739564 & 60.4807172537 & 780663.5282 & 43466.75418 & 0.9021455 & & 0155.3435526 & 48.70452355 & 20.930212 \\
\hline 9 & 9821.44315 & 65291.62604 & 250 & 287.9419448 & 0.4507868921 & 28.183341 & & 31.19569508 & 9.5696515 & 70.5607312205 & 580660.13574 & 43467.26116 & 0.9022235 & & 0 155.3441855 & 48.70331238 & 20.93105441 \\
\hline 10 & 9795.973428 & 65290.43035 & 250 & 287.8337049 & 0.45057513 & -1527.608883 & & 31.195549404 & 10.765200 & 80.6407150941 & 180656.74665 & 43467.76698 & 0.9023015 & & 0155.3448182 & 48.70210167 & 20.93189647 \\
\hline 11 : & 9770.51328 & 65289.2348 & 250 & 287.7255606 & 0.4503635773 & -1527.034933 & & 31.195403869 & 11.960604 & 90.720668901 & 80653.36092 & 43468.27164 & 0.9023794 & & 0155.3454505 & 48.7008914 & 20.93273817 \\
\hline 13 & 9719.621673 & 65286.84414 & 250 & 287.5095584 & 0.4499410985 & 525.888553 & & 31.195113227 & 14.350976 & 110.8804864211 & 180646.59956 & 43469.2775 & 0.9025349 & & 0155.3467144 & 8.69847221 & 20.9344205 \\
\hline 14 & 9694.190197 & 65285.64902 & 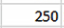 & 287.40 & 0.4497301719 & 25.316121 & & 31.194968118 & 15.545944 & 373 & 380643.2239 & 43469 & 125 & & 459 & 6329 & 113 \\
\hline 15 & 9668.768262 & 65284.45406 & 250 & 287.2 & 0.4495194534 & 24.744195 & & 3 1.194823151 & 16.740767 & 131.040183993 & 80639.85159 & .27873 & 0.9026900 & & 0 155.3479771 & .69605481 & 20.9361014 \\
\hline 16 & 9643.355858 & 65283.25923 & 250 & 287.1862693 & 0.4493089427 & 1524.172773 & & 31.194678325 & 17.935446 & 141.119987864 & 80636.48263 & 43470.77762 & 0.9027674 & & 0 155.3486081 & 48.69484679 & 20.93694132 \\
\hline
\end{tabular}

Figure 10. An example of descent trajectory data modelled with BADA

a)

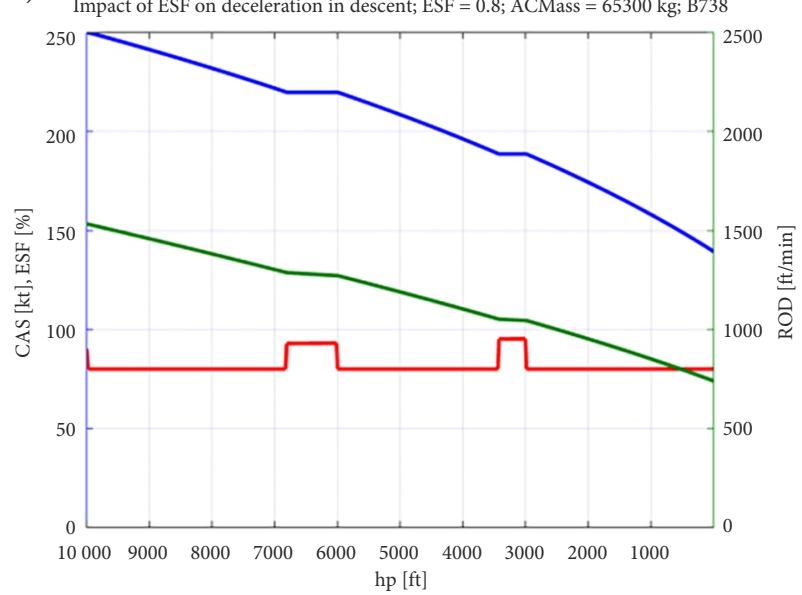

b)

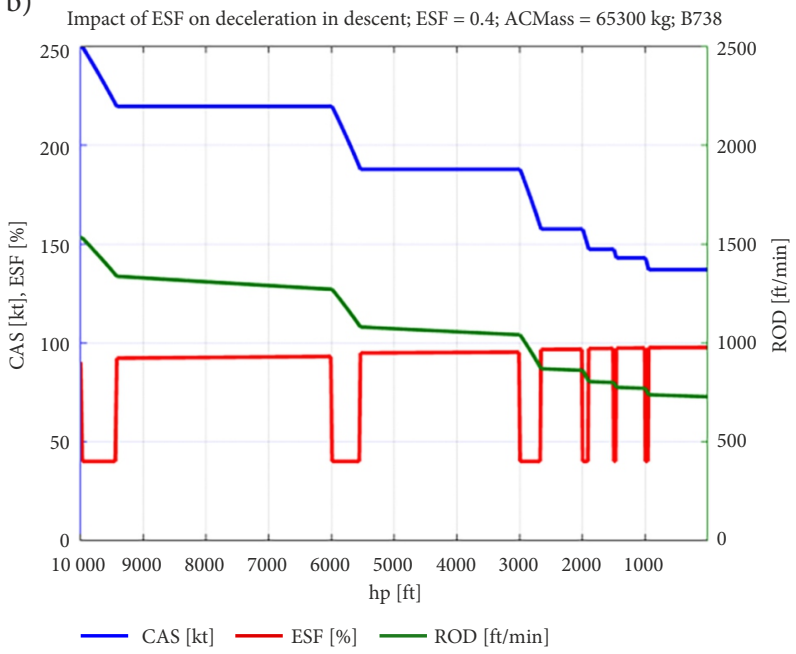

Figure 11. Impact of ESF on deceleration for ESF $($ ACMass $=65300 \mathrm{~kg}): \mathrm{a}-\mathrm{ESF}=0.8 ; \mathrm{b}-\mathrm{ESF}=0.4$

After detailed analysis partially published in paper by Gričová (2016) and Glaser-Opitz et al. (2016), it was found out that according to designed descent trajectory and descent ARPM for KSC, the most suitable value for ESF will be 0.6 (Figure 12). This value for deceleration simulates the most similar procedure to the real one. In the end, it represents the optimal amount of fuel burned during descent phase in comparison with smaller ESF values. Greater ESF values cause the violation of designed speed schedule. Also more rapid deceleration caused by smaller ESF values increases time to descend to the RWY

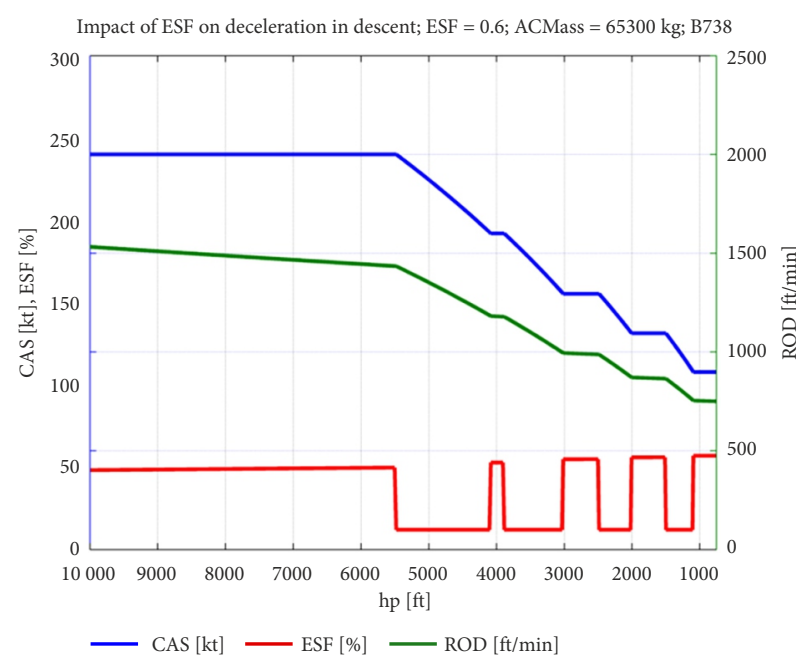

Figure 12. Deceleration in descent on KSC based on designed procedure from the merging point

threshold. Figure 12 depicts simulation of descent with constant $3^{\circ}$ gradient angle, designed based on latest requirement on descent trajectory.

\subsection{BADA model validation tool}

Implementation of BADA model into the Landing System algorithms requires validation, to see if the aircraft is behaving as it is supposed to. The validation tool (Figure 13) was used to validate all output parameters including aircraft weight or fuel flow and other important parameters in all phases of flight and under various conditions we can encounter designing the Landing System using BADA model. BADA model validation tool is depicted on Figure 13.

Real time simulation is based on time integration method with step frequency set to $1 \mathrm{~Hz}$. Figure 13 is showing only 6 graphs for BADA output parameters, with an option to choose more of them, specifically: $\mathrm{Hp}$ (geopotential altitude); ROCD; CAS/TAS; GRAD; FFlow (aircraft fuel flow); FWeight (weight of fuel burned from the start of the simulation); ACMass (changed due to fuel burned during the flight); Distance (travelled by aircraft from the start of simulation); Air temperature (time change of air temperature from the simulation start); Air pressure (time change of air pressure from the simulation start); Air density (time change of air density from the simulation start); Thrust (actual engine thrust); Drag (actual aircraft drag); Mach (hach speed); actual ESF. 
a)

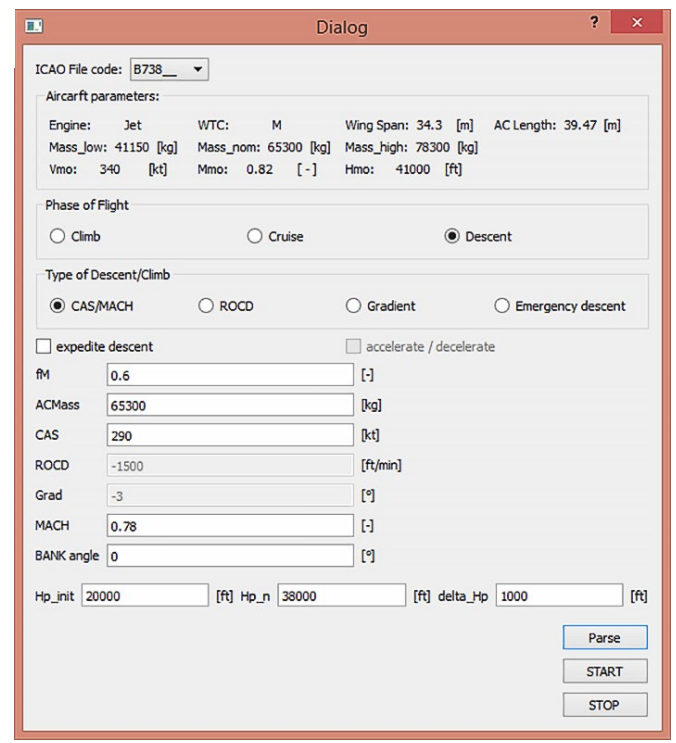

b)

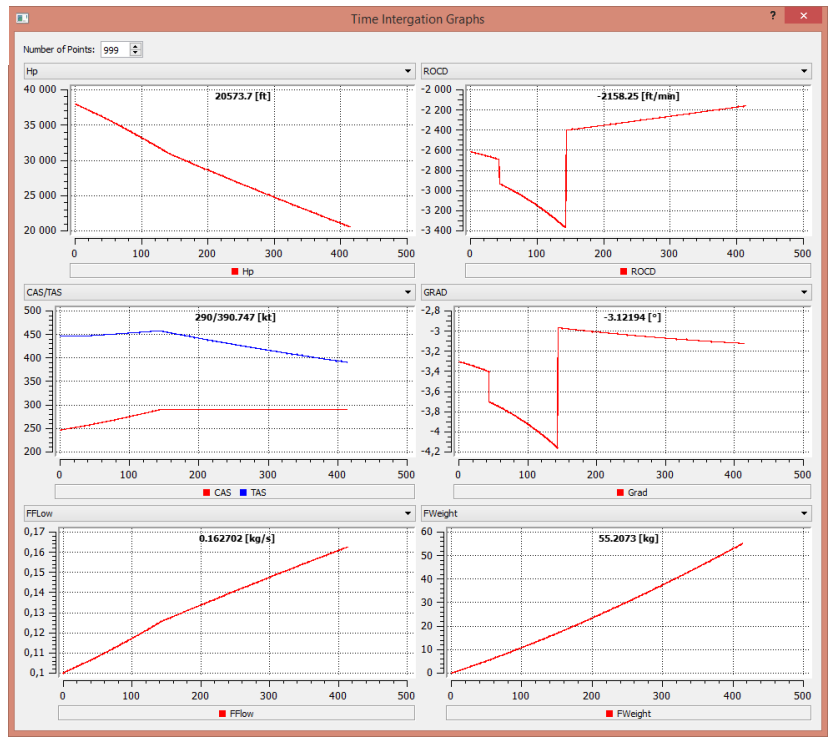

Figure 13. BADA model validation tool (a) with time integration output (b)

\section{Landing System prototype implementation}

The primary reason of CFIT is a lack of situational awareness of surrounding terrain in combination with poor visibility or other additional factors. Today pilots must rely mostly on paper, or electronic charts to understand the relationship between terrain and the own ship, estimate their position by finding their location to nearby radio navigation aids, and after all mentally project the relative location onto the charts. This increases the pilots overall workload especially during climb, descent and landing phase of flight. Moving maps in combination with glass cockpit brings an improvement thanks to the simpler representation of aircraft position. On the other hand, also require additional attention diverted from primary flight instruments. Synthetic vision system that combines a terrain database, position sensor information, computing platform and display, allows pilot very natural and intuitive view of terrain features ahead. Addition of such system could dramatically reduce the number of CFIT accidents by increasing the pilot situational awareness (Wenger 2007).

The Prediction Landing System was developed using Qt programming environment (Qt 2016). Throughout the development process it was tested using FSX flight simulator as a source of all data to test the Landing System functionality. The whole simulation and development process was focused on KSC and its surroundings. This airport was chosen due to its vicinity and simplicity for pointing out the main advantages of such system. To successful design of descend and landing procedure, we needed more information than is usually publicly available. Thanks to project participants familiarity with its design and data availability for KSC, we were able to gather sufficient information. Descend procedure for this airport was designed in cooperation with professional pilots, tak- ing advantage of his experience with such procedures. We used the data to design airline descend speed profile and procedure. Data sent to Landing System for calculation are updated every $1 \mathrm{~s}$.

Figure 14 illustrates used digital terrain database for developed Landing System and for its testing procedures. The red line depicts the RWY 01/19 in relation to surrounding terrain. Greater precision of Landing System with this approach could be achieved using terrain database with finer resolution. Green line illustrates the final part of modelled descent trajectory according to the terrain database. The system operates in two basic modes: (1) flight mode, (2) approach mode. Flight mode is active throughout the cruise phase of flight and when the aircraft is not within the terrain database in airport surrounding. In other words, all the time when approach mode is not available. An example of flight mode is shown in Figure 15.

When the Landing System is in flight mode, many of its features are not available because there are no data to be calculated from, like vertical speed, deviation from glideslope or relative bearing to the runway threshold. The trajectory displayed is based only on measurement from RA with $1 \mathrm{~Hz}$ frequency, displaying the height of the aircraft over the unknown terrain (AGL). Nevertheless, even such simple information can be very useful as you can foresee the terrain closure just by looking at the change in radar altitude in time to prevent the CFIT. More information about the aircraft attitude, trajectory, terrain and airport can be derived in approach mode. Approach mode is active during the descent and go-around phase of flight. That means, when the aircraft is within the terrain database in airport surrounding. This mode is activated automatically. An example of approach mode is shown in Figure 16. 
Altitude displayed in approach mode is calculated and depicted as an altitude AMSL. The aircraft altitude is calculated from RA measurements and added to the terrain elevation from the terrain database. The knowledge of air- craft position, terrain in its vicinity and exact flight path for descent to the RWY enables the terrain prediction. This increases the pilot situational awareness and reduces the CFIT probability.

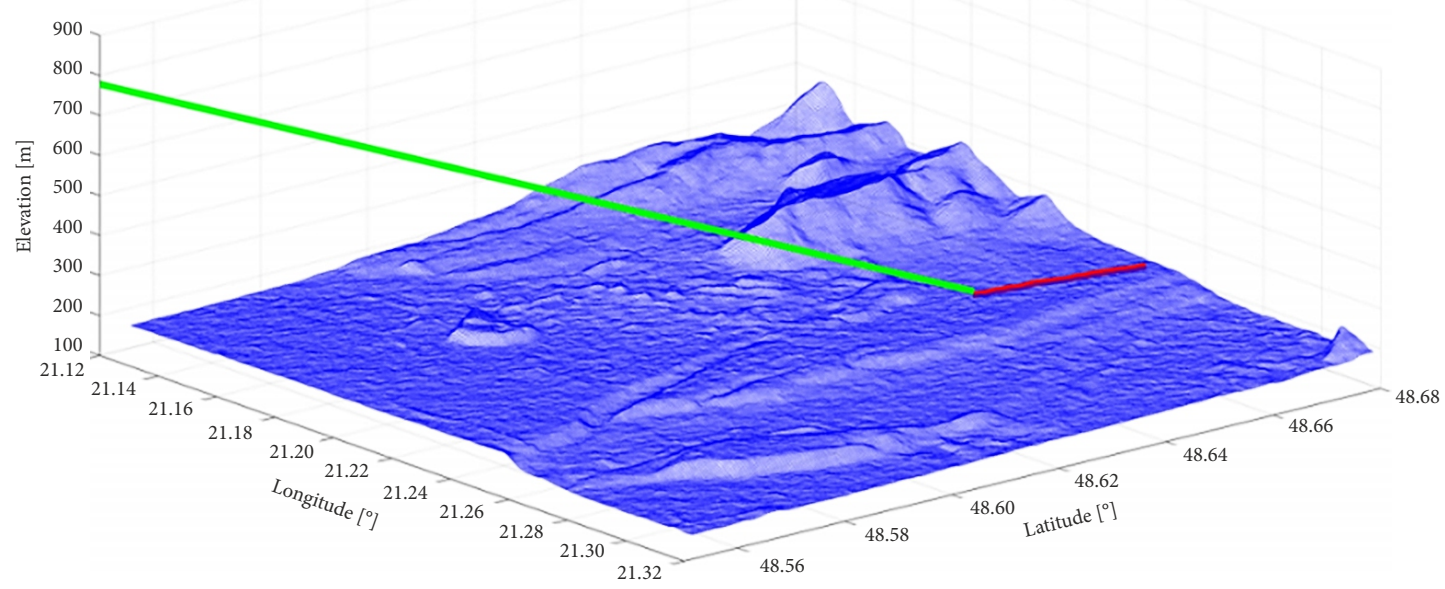

Figure 14. KSC digital terrain database

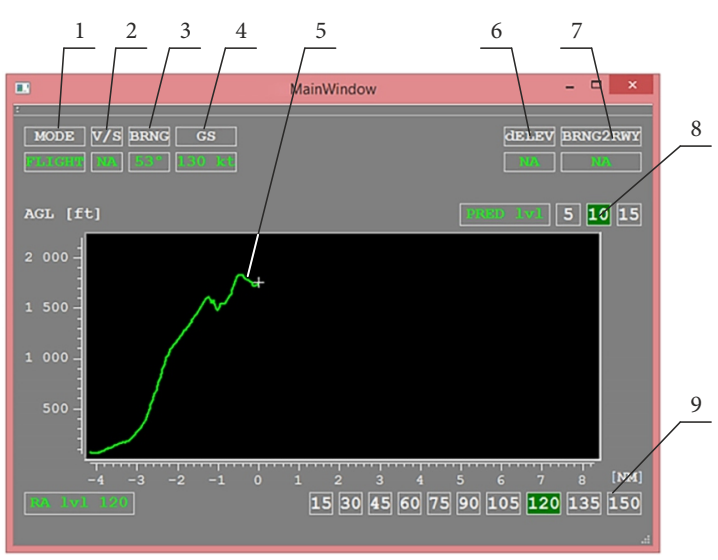

Figure 15. Landing System prototype (flight mode): 1 - active mode indication (currently is active flight mode); 2 - actual aircraft vertical speed (calculated based on last two position recorded); 3 - actual bearing (bearing calculated from last two aircraft positions); 4 - actual ground speed (calculated based on last two aircraft position and distance between these points on the ground using Vincenty formula (MTL 2016)); 5 - flown trajectory according to data received by Landing System from RA sensors; 6 - deviation from desired altitude according to designed descent trajectory; 7 - relative bearing to the runway threshold; 8 - level of trajectory prediction [seconds ahead]; 9 - level of displayed flown trajectory (in numbers of data received, $120=120 \mathrm{~s}$ in history)

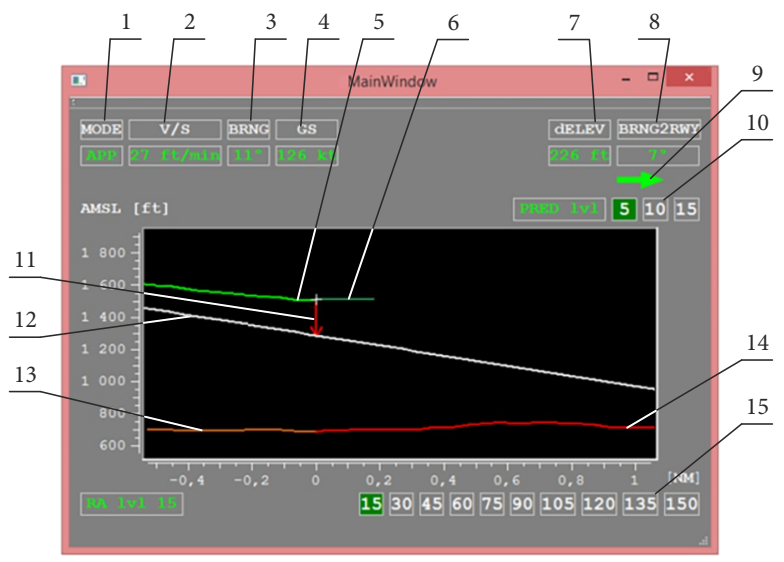

Figure 16. Landing System prototype (approach mode): 1 - active mode indication (currently is active approach mode); 2 - actual aircraft vertical speed (calculated based on last two position recorded); 3 - actual bearing (bearing calculated from last two aircraft positions); 4 - actual ground speed (calculated based on last two aircraft position and distance between these points on the ground using Vincenty formula (MTL 2016)); 5 - flown trajectory according to data received by Landing System from RA sensors; 6 - aircraft trajectory prediction based on BADA model; 7 - deviation from desired altitude according to designed descent trajectory; 8 - relative bearing to the runway threshold; 9 - arrow depicting direction in which the aircraft should move in horizontal plane according to relative bearing to runway threshold; 10 - level of trajectory prediction [seconds ahead]; 11 - graphical visualization of difference between actual and desired descent trajectory; 12 - designed descend trajectory used BADA model for descend trajectory modelling from merging point; 13 - terrain elevation that the aircraft flown over according to the terrain database; 14 - predicted terrain according to defined descent trajectory and terrain database; 15 - level of displayed flown trajectory (in numbers of data received, $15=15 \mathrm{~s}$ in history) 


\section{Flight simulator flight test}

Landing System makes use of server application designed primary for DLCSim (Glaser-Opitz, H., Glaser-Opitz, L. 2015a). Therefore the data that are sent from flight simulator to server application were chosen accordingly. Either way, the Landing System can make use of most of them.

All selected data are transferred via client application (FSXtoDLCSim) directly from flight simulator in $1 \mathrm{~s}$ interval (subjected to change). These data are updated immediately when they are significantly changed. The FSXtoDLCSim GUI is depicted in Figure 17 together with console window displaying part of the information sent from flight simulator to make sure that data sent are valid. Parameter selection enables to test missing data and validate the Landing System functionality for various combinations of available/missing on-board navigation data. This also enables to test ATCo reaction when controlling the airspace while the pilot is flying the flight simulator. The whole communication between all client applications, including Landing System is based on server-client communication using TCP/IP protocol (Glaser-Opitz et al. 2015).

Flight simulator served as a testing device for the Landing System functionality. The Landing System worked as expected in both flight and approach modes as illustrated in Figure 18 respectively.

Test flight was performed as an approach procedure to KSC, following by a go-around procedure to test the
Landing System behaviour in various cases. The test included approaching airport runway threshold from both sides to test switching between both operational modes.

\section{Conclusions}

This paper describes a method of flight safety enhancement as one of the most critical feature in aviation.

For flight safety and pilot situation awareness enhancement was chosen method based on TRN with focus on approach and landing phase of flight. This method was verified using $M A T L A B^{\circledR}$ with different types of test flight trajectories and created terrain elevation database. The main goal was to find a way to replace standard glideslope information (e.g. from ILS) requiring the airport ground infrastructure.

Within the development of the Landing System, the traditional glideslope was replaced with modelled descent trajectory modelled with EUROCONTROL BADA performance model. For the purpose of the modelling and simulations, BADA validation tool was developed.

All simulations were created for KSC as CDA procedures, based on real world airline data in compliance with i4D trajectory and proposed merging point. Based on mentioned models and simulations, Landing System prototype was developed, with BADA model based trajectory prediction capability. For testing and further research activities was developed server/client communication interface that enables developed Landing System prototype to communicate with flight simulator and with DLCSim.
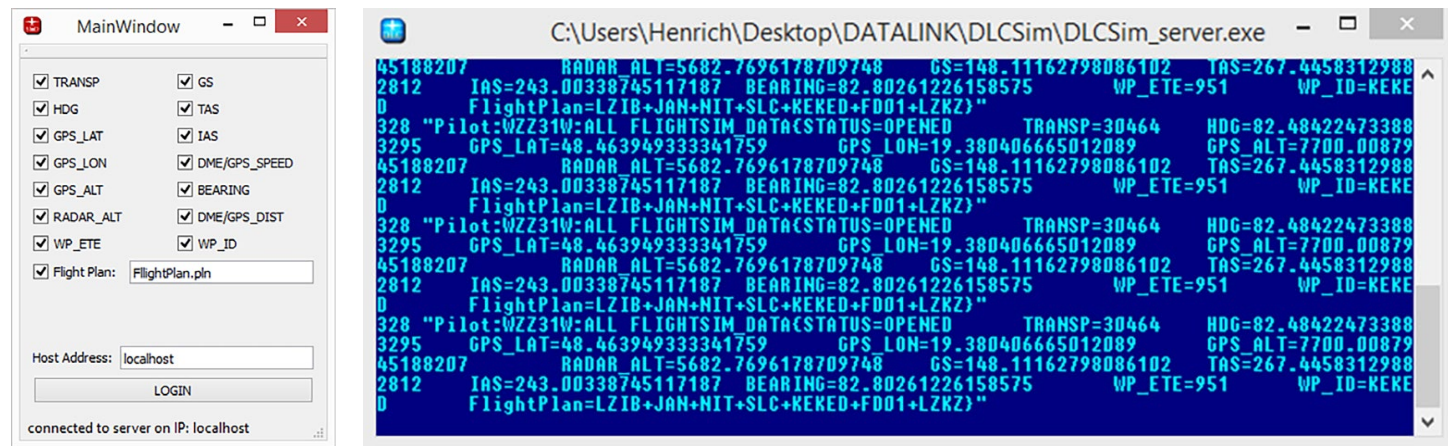

Figure 17. DLCSim data export interface (FSXtoDLCSim) (Glaser-Opitz et al. 2015)
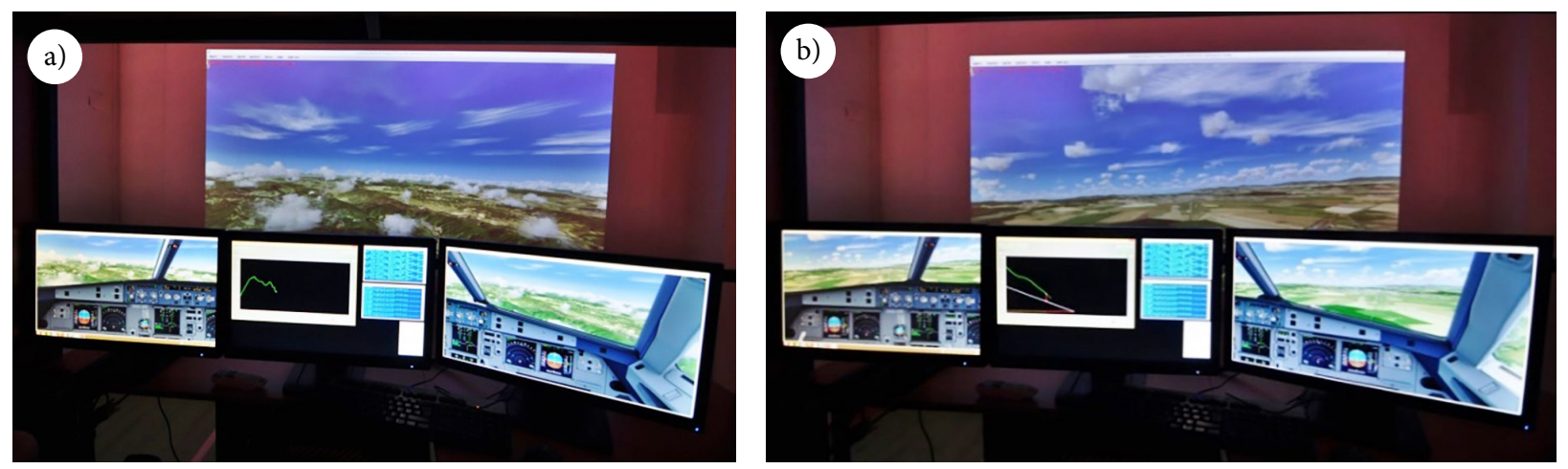

Figure 18. Landing System simulation on flight simulator: $\mathrm{a}$ - flight mode; $\mathrm{b}$ - approach mode 
Enhanced interaction between flight simulator, Landing System and DLCSim enables future research to focus on pilot or ATCo workload during any phase of flight using future avionic systems already available on modern aircraft as well as in research stage.

\section{Disclosure statement}

Authors do not have any competing financial, professional or personal interests from other parties.

\section{References}

EUROCONTROL. 2015. User Manual for the Base of Aircraft Data (BADA). Revision 3.13. European Organisation for the Safety of Air Navigation (EUROCONTROL).

EUROCONTROL. 2014. Coverage of 2013 European Air Traffic for the Base of Aircraft Data (BADA). Revision 3.12. European Organisation for the Safety of Air Navigation (EUROCONTROL).

EUROCONTROL. 1987. Aircraft Modelling Standards for Future ATC Systems. European Organisation for the Safety of Air Navigation (EUROCONTROL).

Gillet, S.; Nuic, A.; Mouillet, V. 2010. Enhancement in realism of ATC simulations by improving aircraft behaviour models, in 29th Digital Avionics Systems Conference, 3-7 October 2010, Salt Lake City, UT, US, 2.D.4-1-2.D.4-13. https://doi.org/10.1109/DASC.2010.5655482

Glaser-Opitz, H.; Glaser-Opitz, L. 2015a. DLCSim: Controllerpilot DataLink Communication Simulator: Version 1.1.0. 39 p. Available from Internet:

https://sourceforge.net/projects/dlcsim/files/Documentation

Glaser-Opitz, H.; Glaser-Opitz, L. 2015b. Evaluation of CPDLC and voice communication during approach phase, in 2015 IEEE/AIAA 34th Digital Avionics Systems Conference (DASC), 13-17 September 2015, Prague, Czech Republic, 2B3-1-2B3-10. https://doi.org/10.1109/DASC.2015.7311363

Glaser-Opitz, H.; Glaser-Opitz, L.; Labun, J. 2015. Data link communication interface with flight simulator in form of a CPDLC, in Proceedings of the International Scientific Conference Modern Safety Technologies in Transportation 2015 (MOSATT 2015), 16-18 September 2015, Zlatá Idka, Slovakia, 63-68.

Glaser-Opitz, H., Gričová, M., Glaser-Opitz, L. 2016. Aircraft trajectory modeling using BADA model, in 5th International Scientific Conference of Ph.D. Students and Young Scientists and Researchers, 12-13 May 2016, Košice, Slovakia, 1-10.

Gričová, M. 2016. BADA Model Analysis for the Purpose of ATM Simulation. Diploma Thesis. Technical University of Košice, Slovakia. $66 \mathrm{p}$.

ICAO. 2013. Performance Based Navigation (PBN) Manual. Doc 9613. International Civil Aviation Organization (ICAO). 4th Edition. 396 p.

Meduna, D. K. 2011. Terrain Relative Navigation for Sensor-Limited Systems with Application to Underwater Vehicles. $\mathrm{PhD}$ Dissertation. Stanford University, Stanford, CA, US. 167 p.
MTL. 2016. Calculate Distance, Bearing and More Between Latitude/Longitude Points. Movable Type Ltd (MTL). Available from Internet:

http://www.movable-type.co.uk/scripts/latlong.html

Qt. 2016. Qt Documentation. Qt Company. Available from Internet: https://www.qt.io

Wenger, J. C. 2007. Development of a Synthetic Vision System for General Aviation. MSc Thesis. University of Iowa, US. 115 p. https://doi.org/10.17077/etd.m3n5d3tn 